\title{
Automated Online Solid Phase Derivatization for Sensitive Quantification of Endogenous S-Nitrosoglutathione and Rapid Capture of Other Low-Molecular-Mass S-Nitrosothiols
}

\author{
Xin Wang ${ }^{*}, \dagger$, Carlos T. Garcia ${ }^{\dagger}$, Guanyu Gong $^{\dagger}$, John S. Wishnok ${ }^{\dagger}$, and Steven R. \\ Tannenbaum ${ }^{*}, \dagger, \ddagger$ \\ tDepartment of Biological Engineering Massachusetts Institute of Technology, Cambridge, \\ Massachusetts 02139, United States \\ FDepartment of Chemistry, Massachusetts Institute of Technology, Cambridge, Massachusetts \\ 02139, United States
}

\section{Abstract}

S-Nitrosothiols (RSNOs) constitute a circulating endogenous reservoir of nitric oxide, and have important biological activities. In this study, an online coupling of solid phase derivatization (SPD) with liquid chromatography-mass spectrometry (LC-MS) was developed and applied in the analysis of low-molecular-mass RSNOs. A derivatizing-reagent-modified polymer monolithic column was prepared and adapted for online SPD-LC-MS. Analytes from the LC auto-sampler flowed through the monolithic column for derivatization, and then directly into the LC-MS for analysis. This integration of the online derivatization, LC separation and MS detection facilitated system automation, allowing rapid, laborsaving, and sensitive detection of RSNOs. SNitrosoglutathione (GSNO) was quantified using this automated online method with good linearity $\left(\mathrm{R}^{2}=0.9994\right)$; the limit of detection was $0.015 \mathrm{nM}$. The online SPD-LC-MS method has been used to determine GSNO levels in mouse samples, $138 \pm 13.2 \mathrm{nM}$ of endogenous GSNO was detected in mouse plasma. Besides, the GSNO concentrations in liver $(64.8 \pm 11.3 \mathrm{pmol} / \mathrm{mg}$ protein), kidney $(47.2 \pm 6.1 \mathrm{pmol} / \mathrm{mg}$ protein), heart $(8.9 \pm 1.8 \mathrm{pmol} / \mathrm{mg}$ protein), muscle $(1.9$ $\pm 0.3 \mathrm{pmol} / \mathrm{mg}$ protein $)$, hippocampus $(5.3 \pm 0.9 \mathrm{pmol} / \mathrm{mg}$ protein $)$, striatum $(6.7 \pm 0.6 \mathrm{pmol} / \mathrm{mg}$ protein), cerebellum ( $31.4 \pm 6.5 \mathrm{pmol} / \mathrm{mg}$ protein), and cortex $(47.9 \pm 4.6 \mathrm{pmol} / \mathrm{mg}$ protein) were also successfully quantified. When the derivatization was performed within 8 minutes, followed by LC-MS detection, samples could be rapidly analyzed compared with the offline manual method. Other low-molecular-mass RSNOs, such as S-nitrosocysteine and S-nitrosocysteinylglycine, were captured by rapid precursor-ion scanning, showing that the proposed method is a potentially powerful tool for capture, identification and quantification of RSNOs in biological samples.

*Corresponding Author: MIT 56-731A, 32 Vassar St, Cambridge, MA, 02139. Tel: (617) 253-3729. Fax: (617) 252-1787. str@mit.edu.

Supporting Information

The Supporting Information is available free of charge via the Internet at http://pubs.acs.org.

Details of the preparation and characterization of GSNO, ${ }^{13} \mathrm{C}_{2},{ }^{15} \mathrm{~N}$-labeled $\mathrm{G} * \mathrm{SNO}$ and derivatizing reagent, additional figures as described in the text (Figure S-1 to Figure S-5), method validation table (Table S-1) and summary of references for endogenous GSNO/RSNO analysis (Table S-2).

Notes

The authors declare no competing financial interest. 


\section{TOC image}

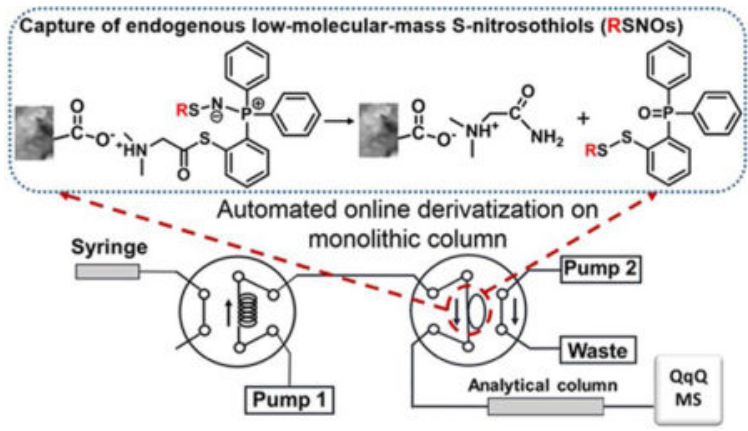

\section{INTRODUCTION}

S-Nitrosothiols (RSNOs) have been identified as the key biologically relevant reaction products induced by reactive nitrogen species, such as nitric oxide (NO•). ${ }^{1} \mathrm{NO} \bullet$ is a critical signaling molecule in living organisms. ${ }^{2}$ In mammals, $\mathrm{NO} \bullet$ is produced from $\mathrm{L}$-arginine by the catalytic action of nitric oxide synthase (NOS) isoforms. ${ }^{3}$ RSNOs have been proposed as important intermediates in $\mathrm{NO} \bullet$ metabolism, storage, as well as mediators in numerous $\mathrm{NO} \bullet$ signaling pathways. ${ }^{4,5}$ Some primary RSNOs such as S-nitrosocysteine (CysNO) and Snitrosoglutathione (GSNO), serve as endogenous NO• carriers and donors. ${ }^{6}$ Despite the considerable number of published studies in the field of RSNOs, quantification of these compounds in biological systems is still a challenge. ${ }^{7,8} \mathrm{~A}$ major obstacle to the reliable measurement of RSNOs is the lack of validated sample preparation procedures capable of assuring RSNO stability during the pre-analytic steps. ${ }^{9,10}$ The chemical lability of RSNOs further complicates this problem. In addition, thiols and inorganic nitrite are usually present at high concentrations in biological matrices, so precautions must be adopted to prevent artifactual formation of RSNOs. ${ }^{11}$

The most widely used methods for RSNOs detection rely mainly on two strategies; one is based on the decomposition of RSNOs followed by detection of released NO.$^{6,12-14}$ In such methods, total decomposition of RSNOs is desired, but also challenging. Besides, these methods preclude identification of multiple RSNO species because the detection is based on the released NO• and total RSNO level will be determined. The other strategy depends on the chemical derivatization of RSNOs; several reagents, e.g., organomercury compounds, 15,16 sulfinic acids, ${ }^{17}$ and phosphines have been evaluated. ${ }^{18-20}$ These reactions, however, are offline with respect to the analytical instruments, and suffer from multiple steps that may degrade the RSNOs, or lead to sensitivity and selectivity issues. Given the limitations of the long sample preparation time in offline methods, it would be valuable to establish an online derivatization and detection approach that is amenable to automation.

Polymer monolith is attractive for sample pretreatment due to its easy preparation, high permeability, large surface area and good control of porosity. ${ }^{21}$ Monolithic capillary columns have been important in solid phase extraction; ${ }^{22}$ their use for derivatization, however, is relatively rare. We chose polymer monolith as a support medium for derivatization for several reasons. On the one hand, monolithic columns are easily 
synthesized in situ using appropriate mixtures of monomers, crosslinkers and porogenic solvents, so diverse polymer monolithic materials can be tailored. ${ }^{23}$ In addition, the biocompatibility of monolith allows the direct analysis of complex biological samples with no manipulations other than dilution or centrifugation, to simplify the entire procedure. ${ }^{24,25}$ In this study, Poly(methacrylic acid-co-ethylene dimethacrylate) (poly(MAA-co-EDMA)) monolith was used as the monolithic support for derivatization because this weak cation exchange monolith can be modified based on ion-exchange and hydrophobic interactions, can cope with biological samples and avoid irreversible protein adsorption. ${ }^{26}$

Here, we describe a novel online solid phase derivatization coupled with a liquid chromatography-mass spectrometry (online SPD-LC-MS) technique that enables rapid discovery and sensitive quantification of endogenous low-molecular-mass RSNOs. The analytes are transferred to the monolith (solid phase) where they are derivatized; so-called solid phase derivatization (SPD). An automated platform with two pumps and two switching valves, and all experimental procedures, were programmed and controlled by the LC-MS workstation. As the main low-molecular-mass RSNO compound in mammalian cells, GSNO was selected as test compound for method optimization and validation. ${ }^{27}$ We also demonstrated that this new online SPD-LC-MS method could capture other endogenous RSNOs, e.g., CysNO and S-nitrosocysteinylglycine (GlyCysNO).

\section{EXPERIMENTAL SECTION}

\section{Chemicals and Reagents}

3-(Trimethoxysilyl) propyl methacrylate, methacrylic acid (MAA), ethylene dimethacrylate (EDMA), toluene, dodecanol, and azobis-(isobutyronitrile) (AIBN) were purchased from Sigma-Aldrich (MO, USA). Fused-silica capillaries with $530 \mu$ m i.d. $\times 720 \mu$ m o.d. were obtained from Polymicro Technologies ${ }^{\mathrm{TM}}$. Acetonitrile (ACN), methanol, acetone and formic acid of LC grade were also from Sigma-Aldrich (MO, USA). Ultra-Pure water was obtained using an in-house purification system. Mercury chloride $\left(\mathrm{HgCl}_{2}\right)$, ethylenediaminetetraacetic acid (EDTA), potassium dihydrogen phosphate $\left(\mathrm{KH}_{2} \mathrm{PO}_{4}\right)$, mPEG-maleimide, sodium nitrite, glutathione (GSH) and ${ }^{13} \mathrm{C}_{2},{ }^{15} \mathrm{~N}$-labeled $\mathrm{G} * \mathrm{SH}$ were from Sigma-Aldrich (MO, USA) and were used without additional purification. Vivaspin 3000 MWCO membrane filters were from Sartorius Stedim (NA, USA). All sample preparations were carried out in the dark at $4{ }^{\circ} \mathrm{C}$ unless otherwise stated. GSNO, ${ }^{13} \mathrm{C}_{2},{ }^{15} \mathrm{~N}$-labeled $\mathrm{G}^{*} \mathrm{SNO}$ and 2-[1-(dimethylamino)ethanethioate]triphenylphosphine (derivatizing reagent) were synthesized as described earlier, ${ }^{18}$ and were confirmed by LC/ESI-HR-MS (see Figure S-1 and Supporting Information for details).

\section{Preparation of poly(MAA-co-EDMA) Monolithic Column}

The inner surface of the fused-silica capillary $(8 \mathrm{~cm} \times 530 \mu \mathrm{m}$ i.d. $)$ was derivatized with 3 (trimethoxysilyl) propyl methacrylate before the polymerization reaction. ${ }^{28}$ The poly(MAAco-EDMA) monolithic column was prepared by a one-step thermally-initiated polymerization. A pre-polymerization solution that consisted of $3.3 \%\left(\mathrm{w} / \mathrm{w}_{\text {total }}\right)$ MAA, $29.1 \%\left(\mathrm{w} / \mathrm{w}_{\text {total }}\right)$ EDMA, $7.6 \%\left(\mathrm{w} / \mathrm{w}_{\text {total }}\right)$ toluene, $59.6 \%\left(\mathrm{w} / \mathrm{w}_{\text {total }}\right)$ dodecanol, and $0.4 \%$ $\left(\mathrm{w} / \mathrm{w}_{\text {total }}\right)$ AIBN was prepared and then drawn into the fused-silica capillary. The reaction 
was performed in an oven at $65^{\circ} \mathrm{C}$ for $16 \mathrm{~h}$. Finally, the capillary column was washed with $\mathrm{ACN}$ to remove the residual reagents.

\section{Offline SPD/LC-MS Method}

The derivatization reaction was carried out in the monolithic column. As shown in Figure 1A, the monolith was connected to a syringe via a Luerlok with the needle replaced by the monolithic column. ${ }^{29}$ The entire experimental sequence involved activation, modification, derivatization, and detection. A syringe infusion pump (Harvard Apparatus, MA, USA) was used to deliver the solution in each step. Methanol and water were used for monolith activation, then $1 \mathrm{~mL}$ of derivatizing reagent $(2 \mu \mathrm{g} / \mathrm{mL})$ was pumped through the monolithic column at $10 \mu \mathrm{L} / \mathrm{min}$ for the in situ functionalization of the poly(MAA-co-EDMA) monolith. The GSNO solution was passed through the derivatizing-reagent-modified monolithic column at $10 \mu \mathrm{L} / \mathrm{min}$, reacting immediately with the reagent. This reaction solution was collected at the end of capillary tube and analyzed by LC-MS.

LC-MS experiments were done on an Agilent 1290 series LC system coupled with an Agilent 6530 Accurate-Mass Quadrupole Time-of-Flight (QTOF) mass spectrometer (Agilent Technologies, CA, USA). The Agilent Eclipse Plus C18 column $(3.5 \mu \mathrm{m} 4.6 \mathrm{~mm} \times$ $100 \mathrm{~mm}$ ) was used for LC-MS analysis. The optimum mobile phases were water with $0.1 \%$ formic acid (A) and ACN with $0.1 \%$ formic acid (B) at a flow rate of $0.5 \mathrm{~mL} / \mathrm{min}$. Gradient elution was applied during the separation (solution B: $30 \%$ for $9 \mathrm{~min}, 30-98 \%$ in $3 \mathrm{~min}$, $98 \%$ for $2 \mathrm{~min}, 30 \%$ for $2 \mathrm{~min}$ ). The column was at room temperature and the injection volume was $10 \mu \mathrm{L}$.

The Agilent Dual AJS ESI source was operated in positive mode and the experimental parameters were as follows: gas temperature, $335^{\circ} \mathrm{C}$; dry gas flow, $8 \mathrm{~L} / \mathrm{min}$; nebulizer, 30 psig; sheath gas temperature, $350^{\circ} \mathrm{C}$; sheath gas flow, $12 \mathrm{~L} / \mathrm{min}$; and capillary voltage, 3.0 $\mathrm{kV}$. The $\mathrm{m} / z$ scan range was from 100 to 1000 with an acquisition rate of $2 \mathrm{spectra} / \mathrm{s}$. The ESI-QTOF was calibrated daily using the standard tuning solution from Agilent. During analysis, the instrument was calibrated in real time with two different reference masses $(\mathrm{m} / \mathrm{z}$ $121.0509,922.0098)$ with constant infusion $(6 \mu \mathrm{L} / \mathrm{min})$. Data were acquired by MassHunter Data Acquisition for Q-TOF B.05.00 (Agilent Technologies, CA, USA) and analyzed by MassHunter Qualitative Analysis B.06.00 (Agilent Technologies, CA, USA).

\section{Automated Online SPD-LC-MS Method}

In order to achieve rapid and sensitive detection of endogenous RSNOs, we next developed the online SPD-LC-MS method by establishing an automated two-pump and two-valve instrumental platform modified from the solid phase microextraction system. ${ }^{30}$ We have changed the flow paths, flow rates and switching sequence of the two valves to realize the online derivatization. The detailed workflows are illustrated in Figure 1B, with valve 1 as the injection valve of the LC system and valve 2 connected to valve 1 with PEEK tubing. The poly(MAA-co-EDMA) monolithic column $(8 \mathrm{~cm} \times 530 \mu \mathrm{m}$ i.d.) was connected at the 3,6 positions of valve 2 . Prior to an experiment, the neat monolithic column was washed with methanol and water (Figure 1B1). Next, $1 \mathrm{~mL}$ of derivatizing reagent $(2 \mu \mathrm{g} / \mathrm{mL})$ was delivered by pump 2 and kept flowing through the monolithic column at $10 \mu \mathrm{L} / \mathrm{min}$ for the 
monolith modification. Meanwhile, the mobile phase (MPS) was driven by pump 1 through the analytical column (Figure 1B2). During analysis, valve 1 was switched for sample injection and valve 2 was also switched from position 2 to position 1 at the same time, so the sample solution from injection loop was driven by pump 1 to flow through the derivatizingreagent-modified monolithic column, to do the online derivatization (Figure 1B3). After switching valve 2 back to position 2, the flow of mobile phase was increased to $0.5 \mathrm{~mL} / \mathrm{min}$ to initiate chromatographic separation (pump 1), while the monolithic column was remodified with the derivatizing reagent (pump 2) until the next injection (Figure 1B4). These procedures were programmed and automatically controlled by the workstation software.

The multiple reaction monitoring (MRM) mode of Triple Quadrupole mass spectrometer (QqQ MS) was used to establish a sensitive and selective quantification method. An Agilent 1100 HPLC system was interfaced to an Agilent 6430 mass spectrometer. Chromatography was based on an Agilent Eclipse Plus C18 column $(3.5 \mu \mathrm{m} 4.6 \mathrm{~mm} \times 100 \mathrm{~mm})$ eluted with a gradient of $0.1 \%$ formic acid in water (A) and $0.1 \%$ formic acid in ACN (B). A specific gradient elution program was set for this online SPD-LC-MC method (solution B: 0-8 min, $30 \%$ at $0.015 \mathrm{~mL} / \mathrm{min} ; 8.01-17 \mathrm{~min}, 30 \%$ at $0.5 \mathrm{~mL} / \mathrm{min} ; 17-20 \mathrm{~min}, 30-98 \%$ at $0.5 \mathrm{~mL} /$ $\min ; 20-22 \mathrm{~min}, 98 \%$ at $0.5 \mathrm{~mL} / \mathrm{min} ; 22.01-25 \mathrm{~min}, 30 \%$ at $0.5 \mathrm{~mL} / \mathrm{min}$ ). The column was maintained at room temperature and the injection volume was $100 \mu \mathrm{L}$ for online derivatization.

The QqQ MS was operated in the positive mode with nitrogen as sheath gas. The capillary voltage was $3000 \mathrm{~V}$. The source gas temperature, gas flow and nebulizer were set at $350^{\circ} \mathrm{C}$, $10 \mathrm{~L} / \mathrm{min}$, and $40 \mathrm{psi}$, respectively. Nitrogen was the collision gas with a collision energy of $10 \mathrm{~V}$. The protonated molecule of derivatized GSNO $(\mathrm{m} / \mathrm{z} 616.2)$ was selected as the precursor ion and the most intensive product ion $(\mathrm{m} / \mathrm{z} 487.1)$ was chosen for the quantification. The quantification ion plus another specific product ion $(\mathrm{m} / \mathrm{z} 309.1)$ were used for confirmation. Data acquisition was carried out by MassHunter Data Acquisition for Triple Quadrupole B.06.00 (Agilent Technologies, CA, USA) and data were analyzed by MassHunter Qualitative Analysis B.06.00 (Agilent Technologies, CA, USA).

\section{Animals}

All mouse experiments were approved by the MIT Committee on Animal Care and were conducted according to the guidelines of Institutional Animal Care and Use Committee (IACUC). 6-Week old female C57BL/6NTac mice were obtained from Taconic Biosciences, Inc. (NY, USA) and housed in a controlled environment at $22 \pm 1{ }^{\circ} \mathrm{C}$ with a relative humidity

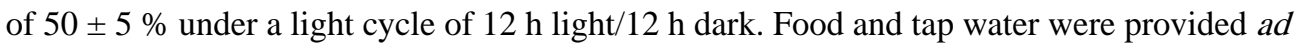
libitum. After adjusting to the environments for 1 week, mice were euthanized by carbon dioxide $\left(\mathrm{CO}_{2}\right)$ inhalation. Euthanasia was achieved by delivering $100 \% \mathrm{CO}_{2}$ from a pressurized system into an enclosed chamber containing the animal, the $\mathrm{CO}_{2}$ flow rate was adjusted to $20 \%$ of chamber volume per minute. Blood samples were collected immediately by cardiocentesis with a $1 \mathrm{~mL}$ syringe and 25 gauge needle. Tissues (liver, kidney, heart, muscle and brain) were then harvested, snap-frozen in liquid nitrogen and stored at $-80^{\circ} \mathrm{C}$ until analysis. 


\section{Mouse Sample Preparation}

Plasma-Blood was collected into $1.5 \mathrm{~mL}$ EDTA-coated Vacutainer tube, and centrifuged at $4{ }^{\circ} \mathrm{C}$ to isolate plasma. $100 \mu \mathrm{L}$ of plasma was spiked with ${ }^{13} \mathrm{C}_{2},{ }^{15} \mathrm{~N}$-labeled $\mathrm{G}^{*} \mathrm{SNO}$ (internal standard) to reach a final concentration of $3 \mathrm{nM}$ after dilution, and immediately treated with $1 \mathrm{mM}$ of mPEG-maleimide in $5 \mathrm{mM} \mathrm{KH}_{2} \mathrm{PO}_{4}$ buffer containing $0.5 \mathrm{mM}$ EDTA $(600 \mu \mathrm{L})$. Sample was kept $15 \mathrm{~min}$ at room temperature for complete blocking by $\mathrm{mPEG}-$ maleimide. Ice-cold ACN $(300 \mu \mathrm{L})$ was added, and precipitated proteins were separated by centrifugation $\left(10000 \times \mathrm{g}, 10 \mathrm{~min}, 4^{\circ} \mathrm{C}\right)$. The supernatant was further ultrafiltered using Vivaspin $3000 \mathrm{Da}$ filter $\left(9000 \times \mathrm{g}, 30 \mathrm{~min}, 4^{\circ} \mathrm{C}\right)$; filtrate solution (low-molecular-weight fraction) was collected and immediately analyzed by the online SPD-LC-MS system. Control GSNO-free plasma was prepared by adding $\mathrm{HgCl}_{2}$ to plasma up to $30 \mathrm{mM}$ to degrade the S-NO bonds. ${ }^{9}$

Tissue sample preparation-Tissue samples were spiked with internal standard $\left({ }^{13} \mathrm{C}_{2},{ }^{15} \mathrm{~N}\right.$-labeled $\left.\mathrm{G} * \mathrm{SNO}\right)$ and immediately mixed with mPEG-maleimide $(1 \mathrm{mM})$ in 5 $\mathrm{mM} \mathrm{KH}{ }_{2} \mathrm{PO}_{4}$ buffer containing $0.5 \mathrm{mM}$ EDTA; typically 1 milliliter buffer per $200 \mathrm{mg}$ of tissue. Samples were then manually homogenized on ice. Lysed samples were kept $15 \mathrm{~min}$ at room temperature to allow complete blocking by mPEG-maleimide, and centrifuged (16 000 $\times \mathrm{g}, 15 \mathrm{~min}, 4^{\circ} \mathrm{C}$ ) to remove cell debris. A $30 \%$ volume of $\mathrm{ACN}$ was added for extraction, followed by filtration using Vivaspin $3000 \mathrm{Da}$ filter $\left(9000 \times \mathrm{g}, 30 \mathrm{~min}, 4^{\circ} \mathrm{C}\right)$. The filtrate solution (low-molecular-weight fraction) of each sample was collected and analyzed by online SPD-LC-MS.

\section{RESULTS AND DISCUSSION}

\section{Mechanism of in-capillary SPD}

Poly(MAA-co-EDMA) monolith was selected as the monolithic support for in-capillary SPD mainly because this weak cation exchange polymer monolith can be easily derivatized with 2-[1-(dimethylamino)ethanethioate]triphenylphosphine at a suitable $\mathrm{pH}$ value (pH 6.0). As show in Figure 2, the derivatizing reagent was extracted and attached to the surface of monolith via ion-exchange interactions in situ between the amino group of the derivatizing reagent and the carboxyl group on the monolith. GSNO solutions were then introduced to the modified monolithic columns for derivatization. Mechanistically, the reaction between GSNO and the derivatizing reagent forms an aza-ylide product. Since the sulfur atom in the reagent is directly bonded to the phenyl ring, the resulting aza-ylide leads to a pseudosulfenamide intermediate which is then attacked by the intramolecular phenylthiolate to yield a disulfide-iminophosphorane structure 1 . This intermediate is further hydrolyzed to produce the phosphoryl-disulfide product 2 (derivatized GSNO). ${ }^{18,31}$

The derivatized GSNO (product 2) was detected by QTOF MS in positive mode (monitored/ expected masses, $\left.m / Z=616.1356 / 616.1341[\mathrm{M}+\mathrm{H}]^{+}\right)$. MS/MS analysis confirmed this identification (Figure 3A). The characteristic peak at $\mathrm{m} / \mathrm{z} 487.0921$ represented the loss of glutamate; while the $\mathrm{m} / \mathrm{z} 309.0517$ signal was the fragment from the derivatizing reagent, and the ion at $m / z 231.0444$ was a specific fragment of Glu-Cys. Based on the accurate mass of the precursor ion and these fragments, the molecule with $\mathrm{m} / \mathrm{z}$ of 616.1356 was determined 
as product 2. To confirm the structure of derivatized GSNO and the mechanism of incapillary SPD, the derivatization was carried out with ${ }^{13} \mathrm{C}_{2},{ }^{15} \mathrm{~N}$-labeled $\mathrm{G}^{*} \mathrm{SNO}$. Compared to the unlabeled products, the precursor ion at $\mathrm{m} / \mathrm{z} 619.1316$ and the characteristic fragment ion of $\mathrm{m} / \mathrm{z} 490.0899$ contained the ${ }^{13} \mathrm{C}_{2},{ }^{15} \mathrm{~N}$-labeling, while the fragment from the derivatizing reagent $(\mathrm{m} / \mathrm{z} 309.0473)$ and fragment of Glu-Cys $(\mathrm{m} / \mathrm{z} 231.0406) \mathrm{did}$ not contain any labeled atoms (Figure 3B).

\section{Optimization of in-capillary SPD Conditions}

An offline SPD/LC-MS method was developed first to demonstrate that in-capillary derivatization could be achieved by the reaction of GSNO with derivatizing reagent via the in situ modified poly(MAA-co-EDMA) monolithic column, as described in the

Experimental Section (Figure 1A). Parameters affecting the derivatization reaction, such as $\mathrm{pH}$, ionic strength and organic solvent content of sample loading solution, were investigated.

As illustrated in Figure 4A, the $\mathrm{pH}$ of the sample loading solution has great influence on derivatization process. The effect of $\mathrm{pH}$ was evaluated in the $\mathrm{pH}$ range of 3.0-8.0 and the highest derivatization efficiency was achieved in the $\mathrm{pH}$ range of 5.0-7.0. This may be related to the interactions between derivatizing reagent and monolithic column. Over the $\mathrm{pH}$ range 5.0-7.0, the derivatizing reagent was protonated and interacted with the ionized carboxyl groups on the monolithic column via ion-exchange interactions, keeping the column well-modified. With a $\mathrm{pH}$ of sample loading solution lower than 5.0 or higher than 7.0, the modified derivatizing reagent was eluted from monolithic column because of the weakened ion-exchange interactions; the derivatization reaction was inhibited in this case. In addition, the eluted derivatizing reagent itself had a strong MS signal in the positive mode, which suppressed the ionization of derivatized GSNO, thus decreasing the derivatization efficiency. The sample loading solution at $\mathrm{pH} 5.0$ was therefore chosen for the in-capillary SPD.

The effect of ionic strength was also studied. Both competing adsorption and kinetic salt effect were observed. When the $\mathrm{KH}_{2} \mathrm{PO}_{4}$ concentration was lower than $15 \mathrm{mM}$, the increase in ionic strength resulted in competing adsorption and decreased the derivatization efficiency. When the $\mathrm{KH}_{2} \mathrm{PO}_{4}$ concentration increased from 15 to $80 \mathrm{mM}$, the kinetic salt effect was more significant and the derivatization efficiency was improved. ${ }^{32,33}$ Finally, 5 $\mathrm{mM}$ of $\mathrm{KH}_{2} \mathrm{PO}_{4}$ was selected for the following experiments (Figure 4B).

Our study indicated that proper addition of ACN would benefit the derivatization reaction, so the ACN content of sample loading solution was optimized systematically for in-capillary SPD. Figure 4C demonstrated that the derivatization efficiency increased significantly with increasing ACN content from $10 \%$ to $30 \%$ (v/v), which was consistent with the previous study. ${ }^{18}$ However, the derivatization efficiency decreased when ACN content exceeded $30 \%$ $(\mathrm{v} / \mathrm{v})$, this may occur because the elution ability of the sample loading solution was enhanced at high ACN concentrations, and caused the elution of modified derivatizing reagent from the monolithic column. Consequently, the optimized sample loading solution was $5 \mathrm{mM}$ of $\mathrm{KH}_{2} \mathrm{PO}_{4}$ at $\mathrm{pH} 5.0$, with the addition of $30 \% \mathrm{ACN}$. 


\section{Automated Online SPD-LC-MS}

Following development of the offline SPD/LC-MS method, we configured an automated online SPD-LC-MS system based on two-pump and two-valve in order to ensure high throughput, sensitivity, and reproducibility for GSNO quantification. The key point of realizing the online derivatization was to set a specific sequence for switching of the two valves, to automatically perform the modification of monolithic column and finish the derivatization reaction, followed by LC-MS analysis. The detailed workflows are illustrated in Figure 1B. The neat poly(MAA-co-EDMA) column was washed by methanol and water, then modified by derivatizing reagent prior to analysis. During online derivatization, the derivatizing reagent reacted with GSNO, so the amounts of derivatizing reagent attached to the monolith surface were reduced. As a result, we needed to re-modify the monolithic column after each injection to achieve satisfactory reproducibility. We successfully added this re-modification step into the automated online system to facilitate continuous injections. As shown in Figure 1B4, LC-MS analysis was conducted after derivatization (pump 1), at the same time, the monolithic column was re-modified by the derivatizing reagent (pump 2) until the next injection. The crucial operating parameters in this system, including the flow rate and reaction time of online derivatization, and concentration of the derivatizing reagent, were optimized to obtain the best detection sensitivity for GSNO.

Figure 5A shows that the flow rate of online derivatization is important. In the beginning, the MS signal increased along with the increase of flow rate and the best signal was acquired at $15 \mu \mathrm{L} / \mathrm{min}$. Decreased MS signals were detected for larger flow rates, because of the dilution effect of MPS, so $15 \mu \mathrm{L} / \mathrm{min}$ was chosen as a preferable flow rate for online derivatization.

Next, the effect of reaction time on GSNO online derivatization was studied from 6 min to 12 min (Figure 5B). Incomplete reaction was observed when the time was shorter than 8 min. Moreover, chromatograms showed peak splitting starting from 9 min of derivatization. Even though the highest MS signal was obtained at $9 \mathrm{~min}$, the derivatization time of $8 \mathrm{~min}$ was eventually used for GSNO quantification considering the chromatographic peak shape and quantitative accuracy.

After each injection, the monolithic column was re-modified by derivatizing reagent during the LC-MS analysis. The concentration of derivatizing reagent was investigated to optimize this re-modification procedure. Signal intensities of the derivatized GSNO were measured while increasing derivatizing reagent concentration from $0.1 \mu \mathrm{g} / \mathrm{mL}$ to $3 \mu \mathrm{g} / \mathrm{mL}$. As illustrated in Figures 5C, the MS signal increased with the increased derivatizing reagent concentration until $1 \mu \mathrm{g} / \mathrm{mL}$, then reached the maximum signal intensity. When this concentration went beyond $1 \mu \mathrm{g} / \mathrm{mL}$, monolith was saturated by the derivatizing reagent in the process of re-modification, thus the MS signal showed no additional increases. A derivatizing reagent concentration of $2 \mu \mathrm{g} / \mathrm{mL}$ was selected for quantification.

\section{Method Validation and Application}

The optimized online method notably increased the sensitivity of GSNO detection compared with the offline mode; this might be ascribed to the online interface of SPD and LC-MS. The flow rate of derivatization reaction was only $15 \mu \mathrm{L} / \mathrm{min}$, while the flow rate of LC-MS 
analysis was $0.5 \mathrm{~mL} / \mathrm{min}$. So after derivatization, the derivatized GSNO was enriched at the analytical column head, and then flushed into the column when the flow rate of mobile phase was increased to $0.5 \mathrm{~mL} / \mathrm{min}$. This "enrichment effect" improved the sensitivity. Typical MRM chromatograms of the derivatized GSNO and derivatized ${ }^{13} \mathrm{C}_{2},{ }^{15} \mathrm{~N}$-labeled G*SNO analyzed by online SPD-LC-MS are illustrated in Figure S-2. The qualitative ion was at $m / z 309.1$, the ions at $m / z 487.1$ and $m / z 490.1$ were selected as quantitative ions for derivatized GSNO and derivatized ${ }^{13} \mathrm{C}_{2},{ }^{15} \mathrm{~N}$-labeled $\mathrm{G}^{*} \mathrm{SNO}$, respectively. So the transitions of $m / z 616.2 \rightarrow 487.1$ and $m / z 619.2 \rightarrow 490.1$ were used for quantification. A matrix-free calibration curve was constructed by plotting the signal intensity versus concentration, relative signal intensity to the internal standard ( $3 \mathrm{nM}$ of ${ }^{13} \mathrm{C}_{2},{ }^{15} \mathrm{~N}$-labeled $\mathrm{G}^{*} \mathrm{SNO}$ ) was used for compensating signal fluctuations during analysis. Satisfactory linearity was obtained in the range of 0.06-30 $\mathrm{nM}$ with a linear coefficient of $\mathrm{R}^{2}=0.9994$ (Figure S-3). The limit of detection (LOD, $\mathrm{S} / \mathrm{N}=3$ ) and limit of quantification (LOQ, $\mathrm{S} / \mathrm{N}=10$ ) were $0.015 \mathrm{nM}$ and $0.054 \mathrm{nM}$, respectively, which is so far the most sensitive method for GSNO quantification. The precision of the method was assessed by measuring the intraday and interday relative standard deviations (RSDs) at three concentration levels; the RSDs were all below $10 \%$ (Table S-1). Therefore, this newly established online SPD-LC-MS method is highly sensitive and quite stable, which is promising for the analysis of GSNO in biological samples.

Finally, the online method was used to analyze GSNO in mouse plasma. Figure 6A shows the MRM chromatogram of derivatized GSNO in the plasma sample. We further diluted the plasma 10 times, and 100 times with optimized sample loading solution in order to accurately quantify the GSNO concentration through the calibration curve (Figure 6B, 6C). As a result, $1.38 \pm 0.132 \mathrm{nM}$ of endogenous GSNO was determined in the 100 times diluted plasma samples, suggesting that the naturally occurring GSNO of $138 \pm 13.2 \mathrm{nM}$ was detected from mouse plasma. Spiking experiments were then performed in the 100 times diluted plasma to assay the accuracy of this method. The recoveries were found to be $90.4 \%$ for the sample spiked with $0.15 \mathrm{nM}$ GSNO, $95.8 \%$ for the sample spiked with $1.5 \mathrm{nM}$ GSNO, and $103.6 \%$ for the sample spiked with $15 \mathrm{nM} \mathrm{GSNO}$ (Table S-1). Due to the matrix-free calibration curve determined in the experiment, recoveries of this method can be used to evaluate the matrix effect. ${ }^{21,34}$ The recoveries were in the range of 90.4 to $103.6 \%$ with RSDs below $10 \%$, indicating that this method did not suffer a negative matrix effect and was applicable to the analysis of endogenous GSNO in complex samples. Additionally, a negative control was prepared by addition of $30 \mathrm{mM}$ (final concentration) $\mathrm{HgCl}_{2}$ to mouse plasma, to degrade the S-NO bonds. ${ }^{9}$ As expected, no GSNO signal was observed after treatment with $\mathrm{HgCl}_{2}$ (Figure 6E).

To further understand the GSNO distribution in 7-week old mice, we measured GSNO levels in several tissues, including the liver, kidney, heart, muscle, and brain (hippocampus, striatum, cerebellum, cortex). The results are illustrated in Figure 7; large amounts of GSNO were detected in liver, kidney, cortex and cerebellum, reflecting previous observations that NOS activity was high in the brain, liver, and kidney of rats; ${ }^{35}$ and NOS expression in the brains of rats and mice occurred mainly in the cortex and cerebellum. ${ }^{36,37}$ Table S-2 also lists the references for endogenous GSNO/RSNOs detection in plasma samples and mouse tissues. 


\section{Capture of Other Endogenous Low-Molecular-Mass RSNOs}

Based on fragmentation analysis, the common qualitative ion $(\mathrm{m} / \mathrm{z} 309.1)$ was produced by breaking the disulfide bond of derivatized GSNO and derivatized G*SNO internal standard (Figure 3). We speculated that other endogenous low-molecular-mass RSNOs could also react with the derivatizing reagent, and, upon CID, produce the $\mathrm{m} / \mathrm{z} 309.1$ product ion. Precursor-ion scanning was consequently conducted with $\mathrm{m} / \mathrm{z} 309.1$ as the product ion over a precursor ion scan window from $m / z$ 100-1000. CysNO and GlyCysNO were detected in mouse samples. Taking CysNO as an example, precursor-ion scanning revealed an intense molecular ion peak at $\mathrm{m} / \mathrm{Z} 430.1$, which matched the theoretical $\mathrm{m} / \mathrm{z}$ value of derivatized CysNO (Figure S-4). MS/MS analysis was then performed in positive mode (Figure S-5). The characteristic product ion of CysNO at $\mathrm{m} / \mathrm{z} 87.1$ and the common product ion at $\mathrm{m} / \mathrm{z}$ 309.1 confirmed the identity, suggesting that the transitions of $\mathrm{m} / z 430.1 \rightarrow 309.1 \mathrm{or} \mathrm{m} / \mathrm{z}$ $430.1 \rightarrow 87.1$ can be used for CysNO quantification in future studies. The LOD was found to be $0.198 \mathrm{nM}$ for CysNO analysis by using the $\mathrm{m} / z 430.1 \rightarrow 309.1$ transition. In analysis of samples from 3 mice, CysNO was detected in tissues including liver, kidney, heart, muscle, hippocampus, striatum, cerebellum, cortex and plasma; GlyCysNO was detected only in liver and cortex. From the above, it is apparent that this automated online SPD-LC-MS method may open a new window for the determination of endogenous low-molecular-mass RSNOs, and which could find additional applications in biological analysis for understanding the crucial roles of RSNOs in cell signaling and homeostasis. ${ }^{38-40}$

\section{CONCLUSIONS}

In the present paper, we have reported a simple, rapid and fully automated online SPD-LCMS technique for the detection and quantification of endogenous GSNO. Polymer monolithic column containing methacrylic acid was prepared in the capillary and connected to the two-pump and two-valve platform for online derivatization. By combining online SPD with LC-MS, the sensitivity of GSNO analysis was greatly improved benefiting from the derivatization and the "enrichment effect" in the new method. We have successfully detected endogenous GSNO in mouse plasma and tissues. Besides, this online SPD-LC-MS method has been extended to the capture of other low-molecular-mass RSNOs, including CysNO and GlyCysNO. Future studies could focus on discovery of changes in RSNOs species and concentrations under specific conditions or disease states, which would actually be of significance and help further our understanding of the physiological roles of RSNOs in various biological systems.

\section{Supplementary Material}

Refer to Web version on PubMed Central for supplementary material.

\section{Acknowledgments}

This work was supported by the National Institutes of Health (US) Grants CA26731 and ES-002109. We thank Uthpala Seneviratne for reviewing our paper and offering helpful advice. 


\section{References}

1. Zhang CH, Biggs TD, Devarie-Baez NO, Shuang SM, Dong C, Xian M. Chem Commun. 2017; 53:11266-11277.

2. Hess DT, Matsumoto A, Kim SO, Marshall HE, Stamler JS. Nat Rev Mol Cell Biol. 2005; 6:150_ 166. [PubMed: 15688001]

3. Forstermann U, Sessa WC. Eur Heart J. 2012; 33:829-837. [PubMed: 21890489]

4. Broniowska KA, Hogg N. Antioxid Redox Signal. 2012; 17:969-980. [PubMed: 22468855]

5. Smith BC, Marietta MA. Curr Opin Chem Biol. 2012; 16:498-506. [PubMed: 23127359]

6. Baldim V, Ismail A, Taladriz-Blanco P, Griveau S, de Oliveira MG, Bedioui F. Anal Chem. 2016; 88:3115-3120. [PubMed: 26892256]

7. Bechtold E, King SB. Antioxid Redox Signal. 2012; 17:981-991. [PubMed: 22356122]

8. Chouchani ET, James AM, Methner C, Pell VR, Prime TA, Erickson BK, Forkink M, Lau GY, Bright TP, Menger KE, Fearnley IM, Krieg T, Murphy MP. J Biol Chem. 2017; 292:14486-14495. [PubMed: 28710281]

9. Bramanti E, Angeli V, Paolicchi A, Pompella A. Life Sci. 2011; 88:126-129. [PubMed: 21047520]

10. Tsikas D, Schmidt M, Bohmer A, Zoerner AA, Gutzki FM, Jordan J. J Chromatogr B. 2013; 927:147-157.

11. Giustarini D, Milzani A, Dalle-Donne I, Rossi R. J Chromatogr B. 2007; 851:124-139.

12. Riccio DA, Nutz ST, Schoenfisch MH. Anal Chem. 2012; 84:851-856. [PubMed: 22201553]

13. Hunter RA, Schoenfisch MH. Anal Chem. 2015; 87:3171-3176. [PubMed: 25714120]

14. Ren H, Coughlin MA, Major TC, Aiello S, Pena AR, Bartlett RH, Meyerhoff ME. Anal Chem. 2015; 87:8067-8072. [PubMed: 26201351]

15. Bramanti E, Angeli V, Mester Z, Pompella A, Paolicchi A, D’Ulivo A. Talanta. 2010; 81:12951299. [PubMed: 20441898]

16. Doulias PT, Greene JL, Greco TM, Tenopoulou M, Seeholzer SH, Dunbrack RL, Ischiropoulos H. Proc Natl Acad Sci U S A. 2010; 107:16958-16963. [PubMed: 20837516]

17. Reeves BD, Hilmer JK, Mellmann L, Hartzheim M, Poffenberger K, Johnson K, Joshi N, Singel DJ, Grieco PA. Tetrahedron Lett. 2013; 54:5707-5710.

18. Seneviratne U, Godoy LC, Wishnok JS, Wogan GN, Tannenbaum SR. J Am Chem Soc. 2013; 135:7693-7704. [PubMed: 23614769]

19. Bechtold E, Reisz JA, Klomsiri C, Tsang AW, Wright MW, Poole LB, Furdui CM, King SB. ACS Chem Biol. 2010; 5:405-414. [PubMed: 20146502]

20. Seneviratne U, Nott A, Bhat VB, Ravindra KC, Wishnok JS, Tsai LH, Tannenbaum SR. Proc Natl Acad Sci U S A. 2016; 113:4152-4157. [PubMed: 27035958]

21. Wang X, Li XJ, Bai Y, Liu HW. Chem Commun. 2015; 51:4615-4618.

22. Masini JC, Svec F. Anal Chim Acta. 2017; 964:24-44. [PubMed: 28351637]

23. Nischang I, Causon TJ. Trac-Trends in Anal Chem. 2016; 75:108-117.

24. Wang X, Ma Q, Li M, Chang CL, Bai Y, Feng YQ, Liu HW. J Chromatogr A. 2013; 1317:121128. [PubMed: 23915641]

25. Arrua RD, Causon TJ, Hilder EF. Analyst. 2012; 137:5179-5189. [PubMed: 22977879]

26. Groarke RJ, Brabazon D. Materials. 2016; 9:446-478.

27. Broniowska KA, Diers AR, Hogg N. Biochim Biophys Acta, Gen Subj. 2013; 1830:3173-3181.

28. Wang X, Lamprou A, Svec F, Bai Y, Liu HW. J Sep Sci. 2016; 39:4544-4548. [PubMed: 27730732]

29. Wang X, Li XJ, Li Z, Zhang YD, Bai Y, Liu HW. Anal Chem. 2014; 86:4739-4747. [PubMed: 24745793]

30. Moliner-Martinez Y, Herraez-Hernandez R, Verdu-Andres J, Molins-Legua C, Campins-Falco P. Trac-Trends in Anal Chem. 2015; 71:205-213.

31. Biggs TD, Weerasinghe L, Park CM, Xian M. Tetrahedron Lett. 2015; 56:2741-2743. [PubMed: 26089576] 
32. Loupy A, Tchoubar B, Astruc D. Chem Rev. 1992; 92:1141-1165.

33. Takahashi K, Tezuka H, Satoh T, Katsumura Y, Watanabe M, Crowell RA, Wishart JF. Chem Lett. 2009; 38:236-237.

34. Van Eeckhaut A, Lanckmans K, Sarre S, Smolders I, Michotte Y. J Chromatogr B. 2009; 877:2198-2207.

35. Thiemermann C, Szabo C, Mitchell JA, Vane JR. Proc Natl Acad Sci U S A. 1993; 90:267-271. [PubMed: 7678341]

36. Bredt DS, Snyder SH. Neuron. 1994; 13:301-313. [PubMed: 7520252]

37. Bhargava HN, Cao YJ. Peptides. 1998; 19:113-117. [PubMed: 9437743]

38. Bak DW, Pizzagalli MD, Weerapana E. ACS Chem Biol. 2017; 12:947-957. [PubMed: 28157297]

39. Frungillo L, Skelly MJ, Loake GJ, Spoel SH, Salgado I. Nat Commun. 2014; 5:5401-5410. [PubMed: 25384398]

40. Rychter M, Gaucher C, Boudier A, Leroy P, Lulek J. Int J Biochem Cell Biol. 2016; 78:156-161. [PubMed: 27394657] 
A

A1

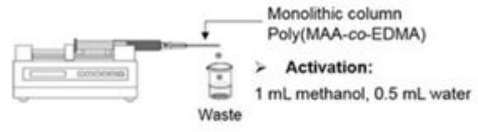

A2

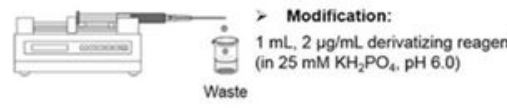

A3

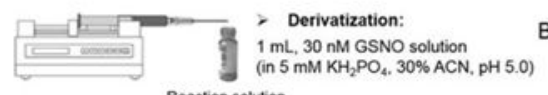
$1 \mathrm{~mL}, 30 \mathrm{NM}$ GSNO solution Reaction solution

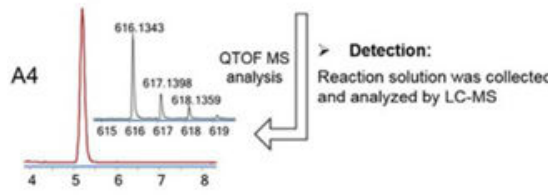

B
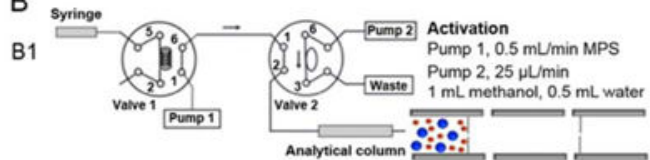

B2

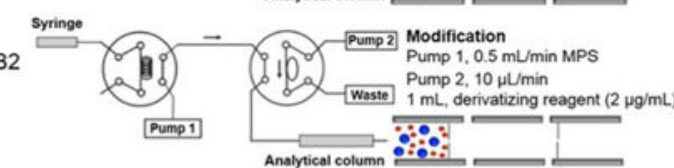

Syringe Pump 2, $10 \mu \mathrm{Lmm}$ Derivatizing reagent $(2 \mu \mathrm{g} / \mathrm{mL})$

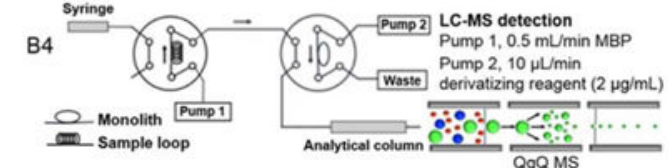

Figure 1.

Experimental set up and configurations of (A) the offline SPD with LC-MS, and (B) automated online SPD-LC-MS for the analysis of GSNO. The experimental sequence involves activation (A1 and B1), modification (A2 and B2), derivatization (A3 and B3), and detection (A4 and B4); operation B is the automated version of operation A. 

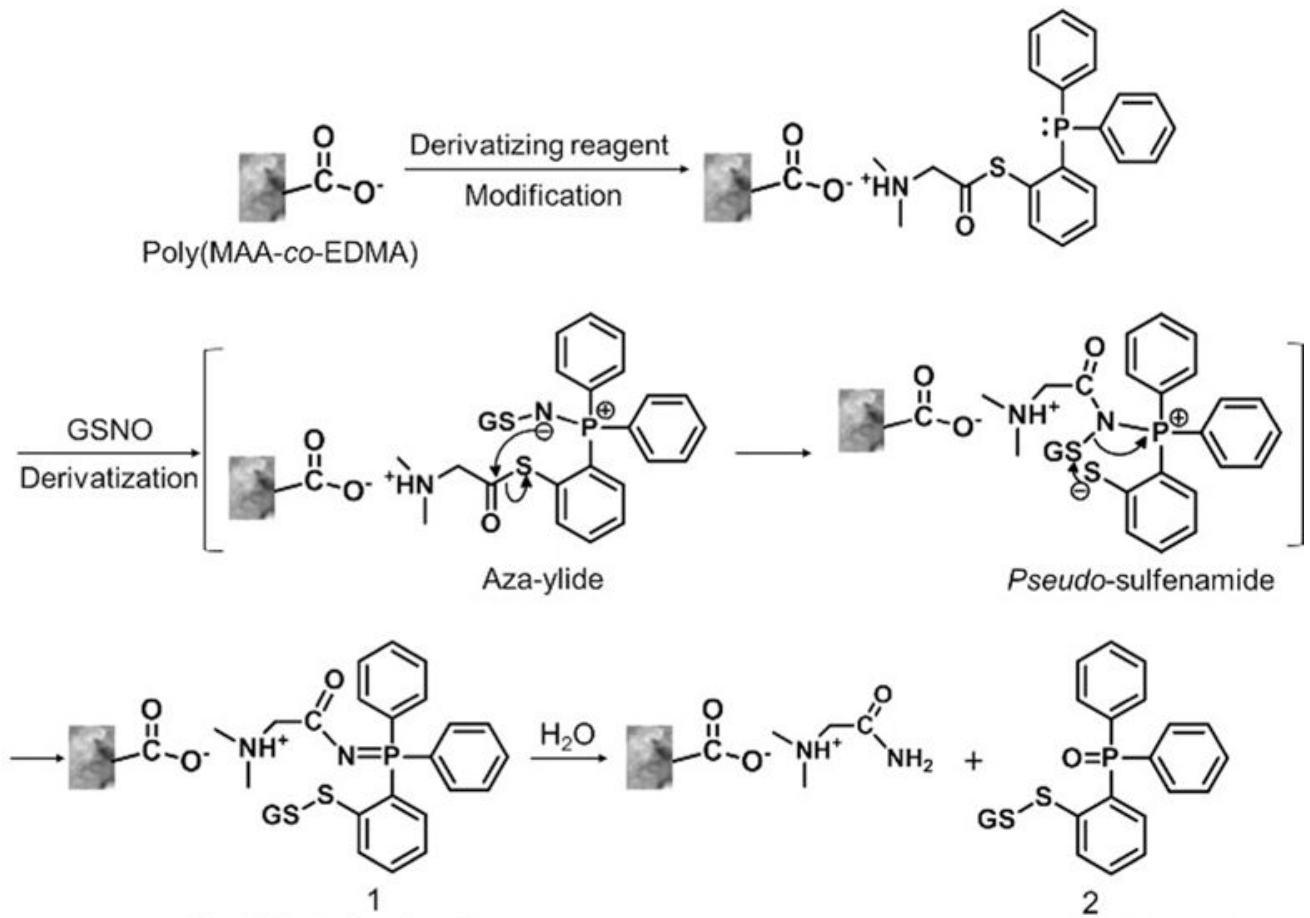

Disulfide-iminophosphorane

Phosphoryl-disulfide product

Figure 2.

Proposed reaction pathways of in-capillary SPD when GSNO reacts with the derivatizingreagent-modified monolithic column. 


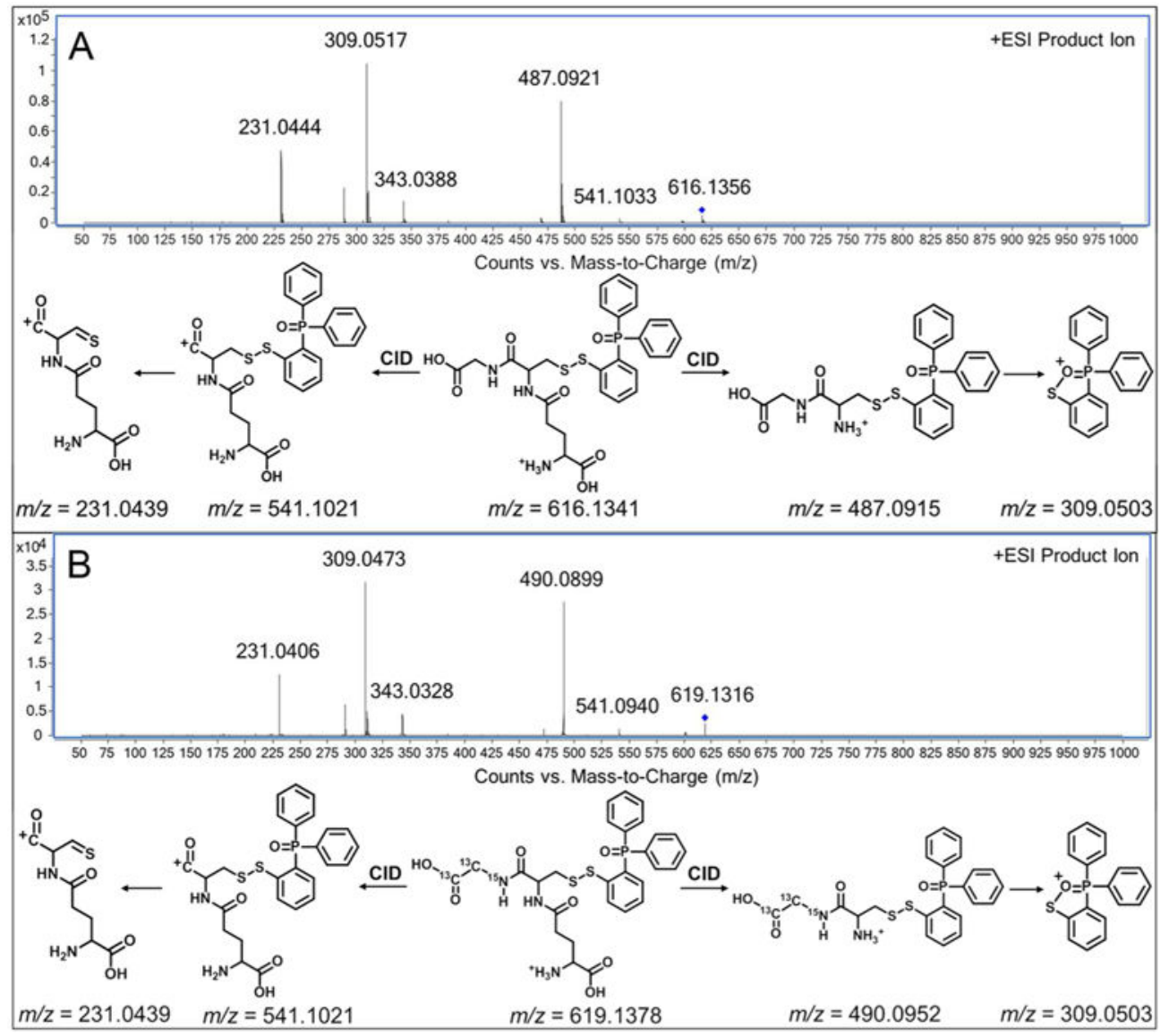

Figure 3.

Tandem mass spectra obtained by QTOF MS in positive mode, and fragment ions interpretations of (A) the derivatized GSNO, and (B) the derivatized ${ }^{13} \mathrm{C}_{2},{ }^{15} \mathrm{~N}$-labeled G*SNO; collision energy: $20 \mathrm{~V}$. 
A

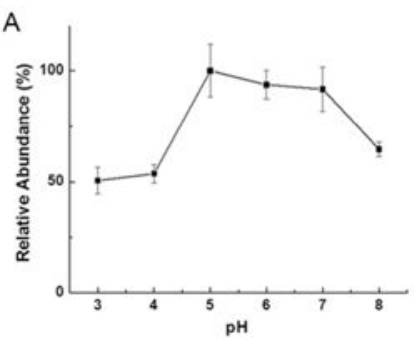

B

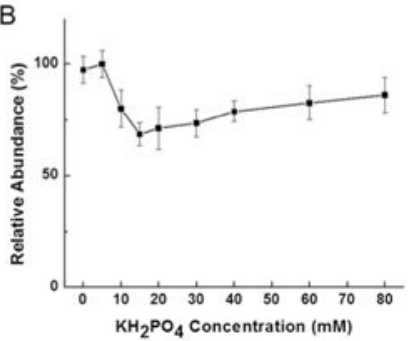

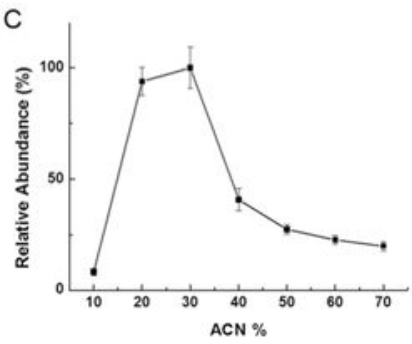

Figure 4.

Effects of (A) the pH value, (B) the ionic strength, and (C) the organic solvent content of the sample loading solution on GSNO derivatization reaction. 

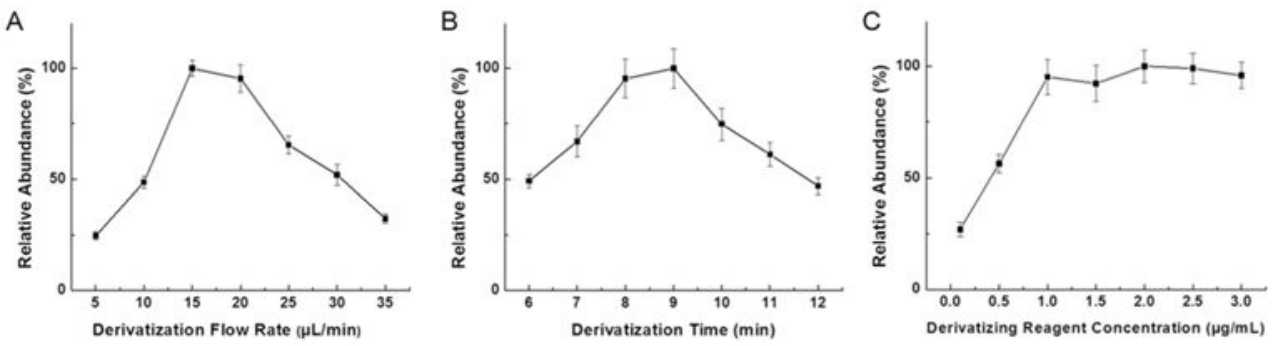

Figure 5.

Optimization of the crucial operating parameters in automated online SPD-LC-MS method for GSNO analysis; (A) flow rate of online derivatization, (B) reaction time of online derivatization, and $(\mathrm{C})$ concentration of the derivatizing reagent used for modification. 


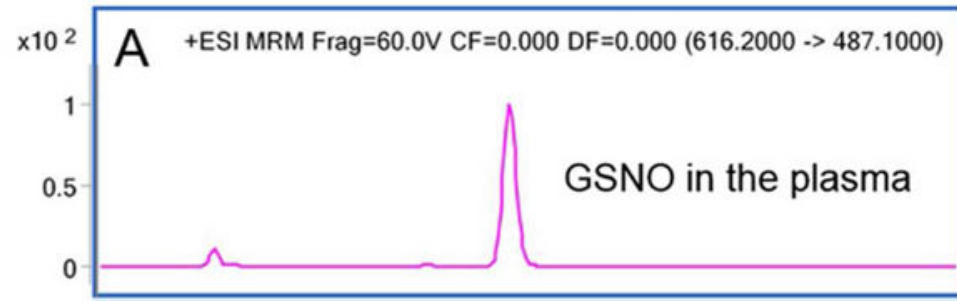

$x 10^{1}$ B +ESI MRM Frag=60.0V $C F=0.000$ DF $=0.000(616.2000 \rightarrow 487.1000)$

2

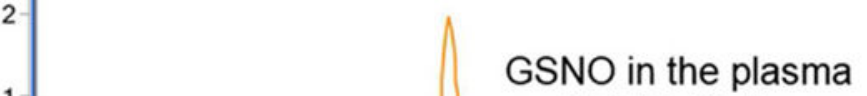

1

0

10 -fold dilution

C +ESI MRM Frag=60.0V CF $=0.000 \mathrm{DF}=0.000(616.2000->487.1000)$

4

GSNO in the plasma

100 -fold dilution

0

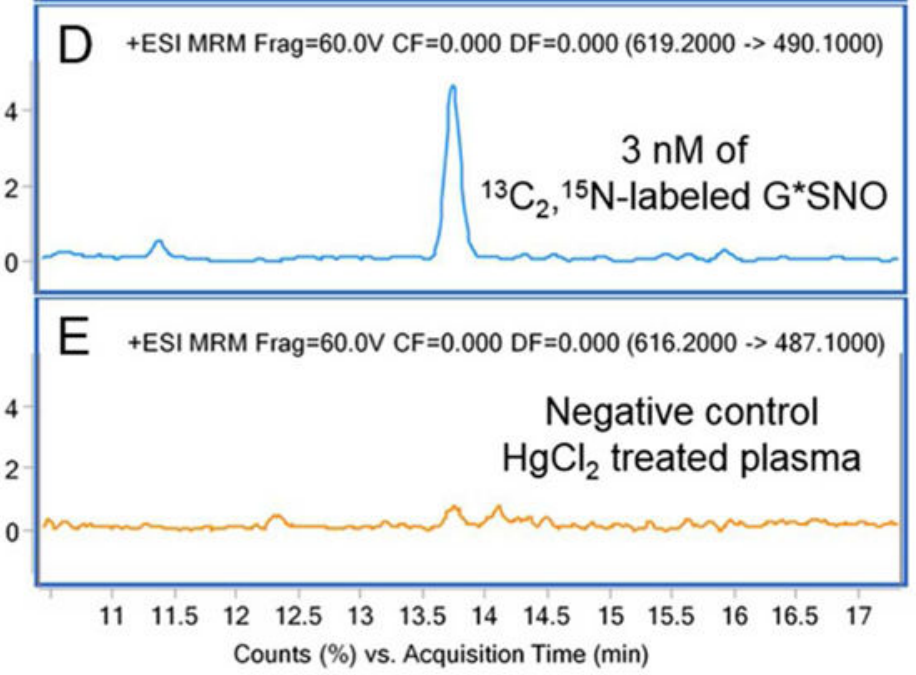

Figure 6.

MRM chromatograms of the derivatized GSNO and derivatized G*SNO internal standard obtained by online SPD-LC-MS method. (A) mouse plasma, (B) 10 times diluted plasma sample, (C) 100 times diluted plasma sample, (D) $3 \mathrm{nM}$ of G*SNO internal standard signal, and (E) $30 \mathrm{mM} \mathrm{HgCl}_{2}$ treated plasma. (Injection volume: $100 \mu \mathrm{L}$ ) 


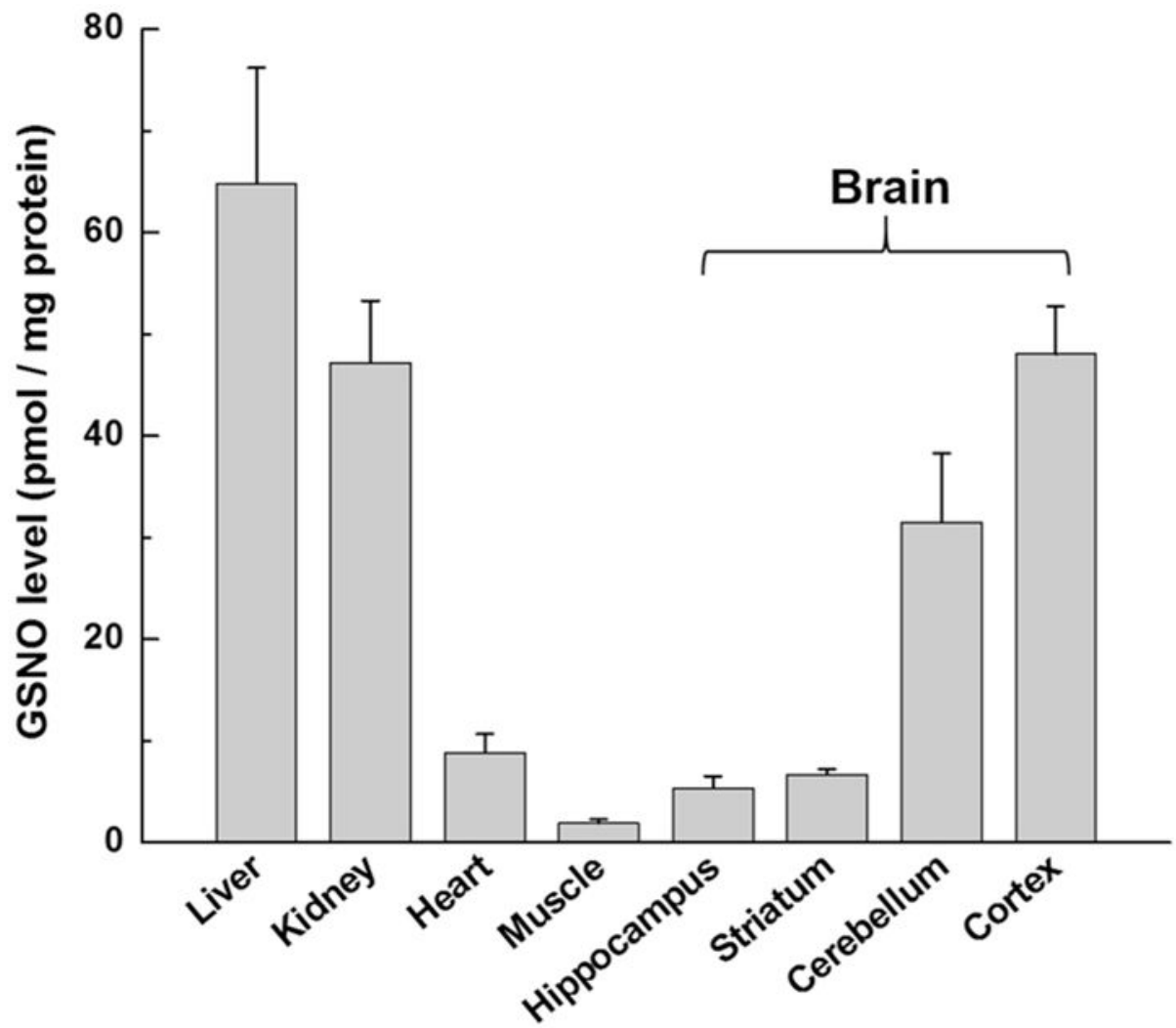

Figure 7.

Profiling of GSNO levels in 7-week old C57BL/6NTac mouse tissues, including the liver, kidney, heart, muscle, and brain (hippocampus, striatum, cerebellum, cortex). For each type of tissue, three biological replicates and three technical replicates for per biological sample were analyzed. 